\title{
ChemComm
}

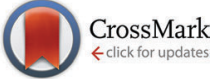

Cite this: Chem. Commun., 2016, 52, 11374

Received 15th August 2016 Accepted 23rd August 2016

DOI: $10.1039 / c 6 c c 06709 c$

www.rsc.org/chemcomm

\section{Creation of new guest accessible space under gas pressure in a flexible MOF: multidimensional insight through combination of in situ techniques $\dagger$}

\author{
Prashant M. Bhatt, Eustina Batisai, Vincent J. Smith and Leonard J. Barbour*
}

\begin{abstract}
Metal-organic frameworks (MOFs) are an important class of porous materials with numerous potential applications. Molecular level understanding of various processes involving MOFs is very important in order to design porous materials with improved properties. Here we describe the elucidation by means of single-crystal X-ray diffraction (SCD) of three different phases of $\mathrm{Zn}_{2}(\mathrm{bdc})_{2}$ (bpy) at different pressures of $\mathrm{CO}_{2}$. Moreover, this is a rare example where new space is created in the structure under gas pressure - space that was not previously occupied by guest molecules in any of the related structures. In addition to in situ SCD and sorption analysis, pressure-gradient differential scanning calorimetry (PG-DSC) provides very useful information about the system. This study represents the first instance where PG-DSC has been used to study the sorption behavior of a flexible porous framework.
\end{abstract}

Phase transformations in solids can be induced by many external stimuli such as heat, light, pressure, etc. In particular, transformations induced by gas pressure have generated much interest: not only do such transformations help us to understand the nature of gas-solid interactions but they can also result in the development of cost-effective industrial processes. ${ }^{1 a}$ Solid-gas interactions generally play an important role in such transformations and the nature of the gas is critical for the transformation to proceed. As one of the early examples, Atwood and co-workers reported polymorphic phase transitions from nonporous to porous phases of organic compounds under gas pressure. ${ }^{1 b, c}$ Metalorganic frameworks (MOFs), ${ }^{2,3}$ one of the most investigated classes of material, are also known to undergo such 'closed' to 'open' structural transformations under gas pressure. The phenomenon is also referred to as 'gate opening' or 'breathing' and this subclass of MOFs is known as 'flexible' or 'soft' MOFs. There has been much interest in the ability of flexible MOFs to undergo

Department of Chemistry and Polymer Science, University of Stellenbosch, Matieland, 7600, South Africa. E-mail: ljb@sun.ac.za; Fax: (+27) 21-808-3360 $\dagger$ Electronic supplementary information (ESI) available: SCD, sorption, PG-DSC, PXRD, CCDC 1429431-1429435. For ESI and crystallographic data in CIF or other electronic format see DOI: 10.1039/c6cc06709c reversible structural changes upon guest removal, addition or exchange $^{3}$ as these features can be used for many potential applications. Recently, Long and co-workers have made use of the flexible nature of a MOF to obtain a better working capacity for methane storage than is exhibited by known rigid frameworks. ${ }^{3 e}$

Chen et al. have reported the preparation of the MOF $\left[\mathrm{Zn}_{2}(\mathrm{bdc})_{2}(\mathrm{bpy})\right] \cdot 2 \mathrm{DMF} \cdot \mathrm{H}_{2} \mathrm{O}\left(\mathbf{1}_{\text {DMF }}\right.$, also referred to as MOF-508a) from the reaction of 1,4-benzenedicarboxylic acid (bdc), 4,4'-bipyridine (bpy) and $\mathrm{Zn}\left(\mathrm{NO}_{3}\right)_{2} \cdot 6 \mathrm{H}_{2} \mathrm{O}$ in DMF/ethanol under solvothermal conditions. ${ }^{4}$ 1DMF is a twofold interpenetrated $3 \mathrm{D}$ MOF with a 2D network of DMF-filled channels. Each framework is based on the zinc paddle-wheel dinuclear secondary building unit (SBU), which propagates in two dimensions via the bdc ligands and in the third dimension by means of bpy pillars. When heated to $120{ }^{\circ} \mathrm{C}$ under dynamic vacuum for 24 hours, 1DMF undergoes a single-crystal to single-crystal transformation to the desolvated form $\left[\mathrm{Zn}_{2}(\mathrm{bdc})_{2}\right.$ (bpy)] (2, previously referred to as MOF-508b). ${ }^{4}$ Although the overall framework connectivity and degree of interpenetration do not change during this transition, the structure of 2 (Fig. 1a) is very different from that of 1DMF. Considerable distortion of the zinc paddle-wheel SBU occurs, with bending in the coordination geometry of the bpy linkers in 2. This results in compression of the framework, with a $16.7 \%$ reduction in the volume of the crystal. A map of the guest-accessible volume in 2 (Fig. 1b) reveals the presence of discrete $66 \AA^{3}$ voids per host formula unit with no permanent channels between them (see ESI $\dagger$ for a comment on void volume calculations). The conversion from 1DMF to 2 is known to be reversible when 2 is soaked in a DMF/ethanol solution for one day. ${ }^{4}$ In the original report describing 1DMF and 2 the authors presumed that 2 undergoes a single phase transition, converting to the gas-included analogue of 1DMF during $\mathrm{CO}_{2}$ sorption. This assumption was based on their sorption isotherm and other information available at that time. ${ }^{4}$

For more insight, we carried out $\mathrm{CO}_{2}$ sorption experiments on 2 at $25{ }^{\circ} \mathrm{C}$ in the pressure range 0-25 bar, as shown in Fig. 2 (top). The amount of gas adsorbed at room temperature and high pressure is comparable to that reported in the literature 


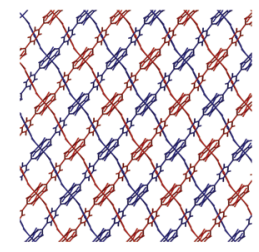

a

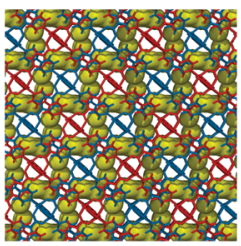

b

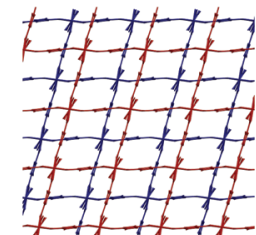

C

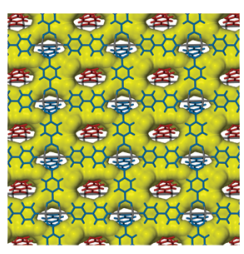

d

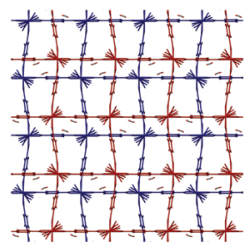

e

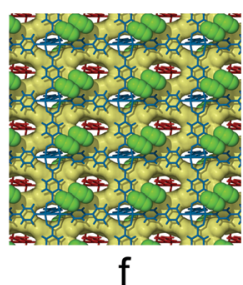

Fig. 1 Perspective views of the twofold interpenetrated networks (colored blue and red) of $\mathbf{2}, \mathbf{1}_{\mathrm{CO}_{2}}$ and $\mathbf{3}_{\mathrm{CO}_{2}}$. (a) $\mathbf{2}$ viewed along the bpy ligand $\mathrm{N} \cdots \mathrm{N}$ axis and (b) OD voids in $\mathbf{2}$ viewed perpendicular to the bpy ligands. (c) $\mathbf{1}_{\mathrm{CO}_{2}}$ viewed along the bpy ligands and (d) guest-accessible $2 \mathrm{D}$ channels in $\mathbf{1}_{\mathrm{CO}_{2}}$. (e) $\mathbf{3}_{\mathrm{CO}_{2}}$ viewed along the bpy ligands and (f) the guestaccessible space corresponding to that of $1_{\mathrm{CO}_{2}}$. The $2 \mathrm{D}$ channel networks in $3 \mathrm{CO}_{2}$ are connected to each other by newly formed guest-accessible channels (not shown but the included $\mathrm{CO}_{2}$ molecules within the new space are shown in green). Note the difference in the coordination geometry of the ligands and the evolution of guest-accessible space concomitant with the structural transformations from OD in $\mathbf{2}$ to $2 \mathrm{D}$ in

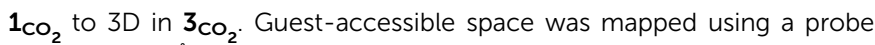
radius of $1.52 \AA$.

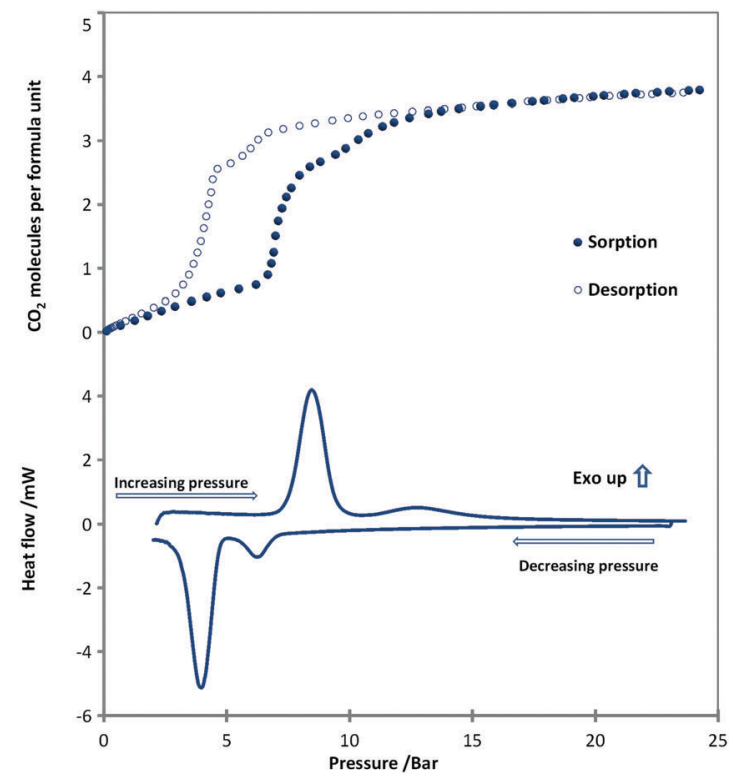

Fig. $2 \mathrm{CO}_{2}$ sorption isotherm for $\left[\mathrm{Zn}_{2}(\mathrm{bdc})_{2}(\mathrm{bpy})\right]$ recorded at $25^{\circ} \mathrm{C}$ (top); inflection points in the isotherm suggest phase transitions (i.e. gateopening events). PG-DSC of $\left[\mathrm{Zn}_{2}(\mathrm{bdc})_{2}(\mathrm{bpy})\right]$ at $25{ }^{\circ} \mathrm{C}$ using a constant pressure gradient of $0.2 \mathrm{bar} \mathrm{min}^{-1}$ in the pressure range 2 to 23 bar of $\mathrm{CO}_{2}$ (bottom).

for low temperature and low pressure. ${ }^{4}$ However, the sorption isotherm at room temperature is much better resolved compared to that recorded at $195 \mathrm{~K}$ and shows two clear inflection points, suggesting that the system undergoes at least two phase transitions under $\mathrm{CO}_{2}$ pressure. It is evident from Fig. 2, that a comparatively small amount of sorption occurs $(\approx 0.8$ moles of $\mathrm{CO}_{2}$ per formula unit) up to 7 bar of $\mathrm{CO}_{2}$ pressure. This sorption trend is consistent with the existence of $66 \AA^{3}$ voids per host forumla unit in 2; each void could conceivably accommodate a maximum of one molecule of $\mathrm{CO}_{2}$. However, after 7 bar the host structure 'opens up' and tends toward taking up an additional two molecules of $\mathrm{CO}_{2}$ per host formula unit. Around 10 bar a second, smaller inflection in the sorption isotherm suggests another structural change in the system to accommodate a further molecule of $\mathrm{CO}_{2}$ per host formula unit. After this point the system slowly reaches saturation in the range of 10 to 25 bar (i.e. 3.82 molecules of $\mathrm{CO}_{2}$ per host formula unit). The desorption cycle also features two inflection points, as well as moderate hysteresis; it is reasonable to assume that the two $\mathrm{CO}_{2}$ pressure-induced 'gate opening' phase transformations are reversed during desorption. The onset pressures for the reverse 'gate closing' phase transitions are approximately 8 and 6 bar - owing to hysteresis, these are somewhat lower values than recorded for the corresponding transitions due to $\mathrm{CO}_{2}$ uptake.

In order to obtain structural insight the pressure-induced phases were investigated crystallographically while subjecting the crystals to controlled $\mathrm{CO}_{2}$ pressures in situ. ${ }^{5}$ Single crystals of $1 \mathrm{DMF}$ were prepared according to the literature procedure ${ }^{4}$ and activated to yield the marginally porous structure 2 (described above). A crystal suitable for SCD analysis was selected and its structures were determined under various pressures of $\mathrm{CO}_{2}$ using an environmental gas cell developed by us. ${ }^{5 a}$ The marginally porous structure 2 with its narrow $0 \mathrm{D}$ voids (Fig. 1b) remains unchanged in the pressure range 0 to 8 bar of $\mathrm{CO}_{2}$ at room temperature $\left(25^{\circ} \mathrm{C}\right)$. When the $\mathrm{CO}_{2}$ pressure is raised to approximately 10 bar, 2 (triclinic, $P \overline{1}$ ) undergoes a single-crystal to single-crystal transformation to yield $\mathbf{1}_{\mathbf{C O}_{2}}$ (also triclinic, $P \overline{1}$ ).

The structure of $\mathbf{1}_{\mathbf{C O}_{2}}$ is similar to that of $\mathbf{1 D M F}$ but with $\mathrm{CO}_{2}$ as the guest instead of DMF. During the transformation from 2 to $\mathbf{1}_{\mathrm{CO}_{2}}$ the grid formed by the dinuclear zinc paddle-wheel SBUs and the bdc linkers distorts only slightly, with the grid angle changing from $72.74(3)^{\circ}$ to $75.57(2)^{\circ}$. However, the most significant structural difference involves the coordination geometry of the bpy ligand, which changes from bent to almost linear. This affects the relative positions of the adjoining grids, which in turn results in changes in the relative positions of the two interpenetrating networks and also the transformation from a 'closed' to an 'open' structure (Fig. 1a and c, respectively). The transformation is thus accompanied by conversion of the narrow $0 \mathrm{D}$ voids of 2 to the comparatively larger guest-accessible channels of $\mathbf{1}_{\mathrm{CO}_{2}}$, which are interconnected in two dimensions (Fig. $1 \mathrm{~b}$ and d, respectively). Since these 2D channels (Channels A) are not linked along the third dimension the structure can be described as possessing segregated 2D channels (Fig. S6b, ESI $\dagger$ ).

Similar to 1DMF, a considerable amount of electron density is apparent in the channels of $\mathbf{1}_{\mathbf{C O}_{2}}$ but the density could not be modelled as individual $\mathrm{CO}_{2}$ molecules. When the $\mathrm{CO}_{2}$ pressure is increased to 20 bar, $\mathbf{1}_{\mathrm{CO}_{2}}$ (triclinic, $P \overline{\mathbf{1}}$ ) converts to a new form 
$3_{\mathrm{CO}_{2}}$ (monoclinic, $P 2_{1} / c$ ) while retaining its crystal singularity. Form $\mathbf{3}_{\mathbf{C O}_{2}}$ can also be obtained by cooling the crystal of $\mathbf{1}_{\mathbf{C O}_{2}}$ to $-20{ }^{\circ} \mathrm{C}$ (the initial $\mathrm{CO}_{2}$ pressure at room temperature was 10 bar but, owing to the small size of the environmental gas cell, the final pressure at $-20{ }^{\circ} \mathrm{C}$ could not be measured). Although the differences in the conformations of the connecting ligands in the structures of $\mathbf{1}_{\mathbf{C O}_{2}}$ and $\mathbf{3}_{\mathbf{C O}_{2}}$ are relatively subtle, these differences have a marked impact on the overall structure and porosity of the material. The largest structural differences between $\mathbf{1}_{\mathrm{CO}_{2}}$ and $\mathbf{3}_{\mathrm{CO}_{2}}$ arise from a change in the shape of the $2 \mathrm{D}$ grid formed by the dinuclear zinc paddle-wheel SBUs and the bdc linkers. In the structure of $\mathbf{1}_{\mathbf{C O}_{2}}$, the grid units resemble a rhombus while in the structure of $\mathbf{3}_{\mathbf{C O}_{2}}$ the linkers are comparatively straight, resembling a square (Fig. $1 \mathrm{c}$ and e, respectively). Another difference between the structures of $\mathbf{1}_{\mathbf{C O}_{2}}$ and $\mathbf{3}_{\mathbf{C O}_{2}}$ is apparent in the torsion angles of the carboxylate groups of the bdc linkers (see Table S2, ESI $\dagger$ ). An even more profound change is observed in the bdc linkers along the crystallographic $a$ axis of $3_{\mathrm{CO}_{2}}$, where the torsion angles change from $8.4(8)^{\circ}$ and $3.8(6)^{\circ}$ to $9.5(3)^{\circ}$ and $8.0(3)^{\circ}$. There is also a moderate change in the dihedral angle between the two pyridyl rings in the bpy linker when the structure changes from $\mathbf{1}_{\mathbf{C O}_{2}}$ to $\mathbf{3}_{\mathbf{C O}_{2}}$, as shown in Fig. 1d, f and Fig. S7 and Animation S1 (ESI + ). Although the individual changes in the abovementioned angles are not very large, in combination with the changes in the grid angles and the other subtle structural changes they ultimately result in the creation of new guest-accessible space in $\mathbf{3}_{\mathbf{C O}_{2}}$ (Channel $\mathrm{B}$, Fig. S6C, ESI $\dagger$ ), which accommodates two extra $\mathrm{CO}_{2}$ molecules for each unique new channel, or an additional $\mathrm{CO}_{2}$ molecule per formula unit (Fig. 1d, f and Fig. S7 and Animation S1, ESI $\dagger$ ). It is worth noting that the region corresponding to that occupied by $\mathrm{CO}_{2}$ in the newly formed channels (Channel B, Fig. S6C, ESI $\dagger$ ) of $\mathbf{3}_{\mathbf{C O}_{2}}$ is unoccupied in $\mathbf{1}_{\mathbf{C O}_{2}} ;$ i.e. no residual electron density is observed in this space. Moreover, the space is not large enough to accommodate any guest molecules in $\mathbf{1}_{\mathbf{C O}_{2}}$. As shown in Fig. S6c (ESI $\dagger$ ), a map of the solvent-accessible surface of $\mathbf{3}_{\mathbf{C O}_{2}}$ shows that the newly formed channels $\mathrm{B}$ interconnect the $2 \mathrm{D}$ channels (Channel A) that are also present in $\mathbf{1}_{\mathbf{C O}_{2}}$.

Although the guest molecules in the larger channels of $\mathbf{3}_{\mathrm{CO}_{2}}$ (at $-20{ }^{\circ} \mathrm{C}$ and 10 bar initial pressure) only yield diffuse electron density distribution patterns in the crystallographic analysis (as was the case for both $\mathbf{1 D M F}$ and $\mathbf{1}_{\mathbf{C O}_{2}}$ ), it was possible to model the $\mathbf{C O}_{2}$ molecules in the smaller channels of $\mathbf{3}_{\mathrm{CO}_{2}}$ (Channels B). It therefore appears that the confined environment of the smaller channels facilitates immobilization and crystallographic modeling of $\mathrm{CO}_{2}$. Site occupancy refinement suggests an occupancy of approximately $80 \%$ for the $\mathrm{CO}_{2}$ molecules in the smaller channels. The geometry of the unique $\mathrm{CO}_{2}$ molecule $(\mathrm{C}=\mathrm{O}$ bond lengths of 1.09(2) and 1.18(2) $\AA$ and a bond angle of $\left.173(2)^{\circ}\right)$ is not ideal but corresponds well with that of structures containing $\mathrm{CO}_{2}$ in the Cambridge Structural Database (CSD) ${ }^{5 c, 6,7}$ Each interconnecting channel contains two symmetry-related $\mathrm{CO}_{2}$ molecules arranged to form an offset dimer that maximizes the interaction between the electron rich oxygen atom $(\delta-)$ and the electron deficient carbon atom $(\delta+)$, as shown in Fig. 3. The intermolecular $\mathrm{C} \cdots \mathrm{O}$ distance between

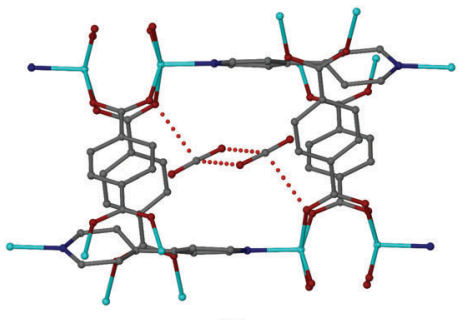

a

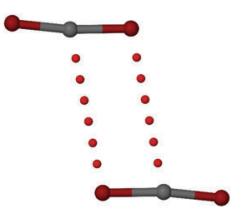

b
Fig. 3 (a) $\mathrm{C}$...O interaction between the $\mathrm{CO}_{2}$ carbon atoms and a carboxylate oxygen atom of the host network in $\mathbf{3}_{\mathrm{CO}_{2}}$; (b) $\mathrm{C} \ldots \mathrm{O}$ interaction in the $\mathrm{CO}_{2}$ dimer.

the two $\mathrm{CO}_{2}$ molecules is 3.13(2) $\AA$; similar $\mathrm{CO}_{2}$ dimers are present in the crystal structure of solid $\mathrm{CO}_{2},{ }^{8}$ as well as in some of the other examples present in the CSD. ${ }^{6}$ The central carbon atom of each $\mathrm{CO}_{2}$ molecule also forms a relatively short $\mathrm{C} \cdots \mathrm{O}$ contact of 3.07(2) $\AA$ with one of the carboxylate oxygen atoms of a bdc linker, as shown in Fig. 3.

There are only a few reports in the literature of nonporous structures that transform to porous phases under gas pressure. $^{1 b, c, 3}$ These processes involve dynamic changes in the host structure in order to accommodate guest molecules, and they seldom occur as single-crystal to single-crystal transformations. Furthermore, in most of these cases a solvate equivalent of the open-framework gas-loaded structure is known, and the nonporous "collapsed" phase is generally prepared by removing the solvent. The collapse of the host framework upon solvent removal is therefore reversible (provided that the gas pressure is sufficient to effect the transformation) and the gas molecules ultimately occupy the space previously templated by the solvent molecules. Indeed, we have observed that this phenomenon occurs for the transformation of solvate 1DMF into nonporous 2 , followed by the transformation of 2 into gasloaded $\mathbf{1}_{\mathbf{C O}_{2}}$. Application of additional $\mathbf{C O}_{\mathbf{2}}$ gas pressure results in transformation to $\mathbf{3}_{\mathrm{CO}_{2}}$, with the creation of new space that had not previously been occupied by guest molecules in any of the known solvated structures involving this framework. To our knowledge, the only other slightly similar example in the literature is the system $\left[\mathrm{M}_{2}\left(\mathrm{O}_{2} \mathrm{CPh}\right)_{4}(\text { pyrazine })\right]_{n}\left(\mathrm{M}=\mathrm{Rh}^{2+}\right.$ or $\mathrm{Cu}^{2+}$ ), widely studied by Takamizawa and others. ${ }^{6,9}$

We considered the desirability of monitoring gas pressureinduced phase transformations or flexibility in MOFs and other porous materials in a time efficient manner using a scanning technique akin to differential scanning calorimetry (DSC), which is commonly employed to observe thermally-induced phase transitions (melting, crystallization, polymorphic conversion, chemical reaction, etc.). Such capability could prove very useful for fast and efficient screening for flexibility and phase transitions of MOFs and other solids in response to gas pressure. Although DSC has already been used to determine the sorption enthalpy for porous materials in a few cases ${ }^{10}$ in all of these examples one or more measurements were made by instantaneous dosing rather than using a scanning approach. Indeed, the limitations of DSC in non-scanning mode for such experiments have been acknowledged in the literature. ${ }^{11}$ DSC has also been 
used in scanning mode to investigate phase transformations of solids under gas pressure either by ramping the temperature at constant gas pressure ${ }^{6}$ or by flowing gas at a constant rate at cryogenic temperatures. ${ }^{11}$ However isothermal DSC analysis while increasing the pressure gradually and at a constant rate has not been realized to date. We therefore developed a method involving pressure-gradient DSC (PG-DSC) and validated the technique using the present MOF. A prototype PG-DSC system was constructed by coupling commercially available modules, including a gas syringe pump (Teledyne ISCO 260D) and a DSC analyser (Setaram $\mu$ DSC7 Evo). In order to increase the gas pressure at a desired constant rate the syringe pump is controlled by a computer running software that was developed by us. We used PG-DSC to study the present system $\left[\mathrm{Zn}_{2}(\mathrm{bdc})_{2}(\mathrm{bpy})\right]$ since this material is now well characterized structurally by in situ crystallography. Fig. 2 (bottom) shows the PG-DSC trace in the range 2-23 bar; two exotherms are observed in the range of 7-10 bar (large) and 11-15 bar (small, broad) during sorption and two endotherms are evident at the shifted pressure range of 7-5 bar (small) and 5-3 bar (large) during desorption. The data are consistent with our results obtained from sorption analysis and in situ SCD.

The PG-DSC peaks are mostly due to the heats of sorption and desorption, combined with a comparatively very small component from the host structural transformations. Therefore the PG-DSC experiments provide valuable information about gate opening/closing onset pressures and gas uptake/release hysteresis in a time-efficient manner. Moreover, it is possible to determine the shifts in these pressures with temperature; the PG-DSC traces for 2 at four different temperatures $(0,15,25$ and $45{ }^{\circ} \mathrm{C}$ ) are shown in Fig. S12 (ESI $\dagger$ ). It is evident that the gate opening pressure and extent of hysteresis increase with temperature. Furthermore, gate opening pressures for a system exposed to different gases can be compared in a rapid and efficient way using PG-DSC. To demonstrate this, PG-DSC experiments were carried out for 2 using $\mathrm{CH}_{4}$ as the test gas and gate-opening occurred in the broad ranges of 30-50 and 60-90 bar at 0 and $25{ }^{\circ} \mathrm{C}$, respectively (See ESI, $\dagger$ Fig. S13 and S14). After validating the PG-DSC technique using the present system we also used the method to detect gas-induced phase transformations for other reported flexible systems (see ESI $\dagger$ ).

In summary, the present work describes the observation of three different structures by SCD at different pressures of $\mathrm{CO}_{2}$ in the system $\mathrm{Zn}_{2}(\mathrm{bdc})_{2}$ (bpy). Moreover, this is a rare example where new guest accessible space is formed under gas pressure: space that was not previously occupied by guest molecules in any of the related structures. The newly-developed technique of PG-DSC was successfully validated using the present system and then also applied to other systems. PG-DSC offers fast and efficient screening of materials for gas-induced phase transformations and efforts to determine useful thermodynamic data from this technique are currently in progress.

The authors thank the National Research Foundation of South Africa for financial support. PB also thanks the Claude Leon Foundation for a fellowship.

\section{Notes and references}

1 (a) J. Tian, S. J. Dalgarno and J. L. Atwood, J. Am. Chem. Soc., 2011, 133, 1399-1404; (b) P. K. Thallapally, B. Peter McGrail, S. J. Dalgarno, H. T. Schaef, J. Tian and J. L. Atwood, Nat. Mater., 2008, 7, 146-150; (c) J. Tian, P. Thallapally, J. Liu, G. J. Exarhos and J. L. Atwood, Chem. Commun., 2011, 47, 701-703.

2 (a) H. Li, M. Eddaoudi, M. O'Keeffe and O. M. Yaghi, Nature, 1999, 402, 276-279; (b) E. D. Bloch, W. L. Queen, R. Krishna, J. M. Zadrozny, C. M. Brown and J. R. Long, Science, 2012, 335, 1606-1610; (c) S. Kitagawa, R. Kitaura and S. Noro, Angew. Chem., Int. Ed., 2004, 43, 2334-2375; (d) Y. Inokuma, S. Yoshioka, J. Ariyoshi, T. Arai, Y. Hitora, K. Takada, S. Matsunaga, K. Rissanen and M. Fujita, Nature, 2013, 495, 461-466; (e) P. Nugent, Y. Belmabkhout, S. D. Burd, A. J. Cairns, R. Luebke, K. Forrest, T. Pham, S. Ma, B. Space, L. Wojtas, M. Eddaoudi and M. J. Zaworotko, Nature, 2013, 495, 80-84; $(f)$ O. K. Farha, A. Özgür Yazaydın, I. Eryazici, C. D. Malliakas, B. G. Hauser, M. G. Kanatzidis, S. T. Nguyen, R. Q. Snurr and J. T. Hupp, Nat. Chem., 2010, 2, 944-948.

3 (a) S. Horike, S. Shimomura and S. Kitagawa, Nat. Chem., 2009, 1, 695-704; (b) C. Serre, F. Millange, C. Thouvenot, M. Noguès, G. Marsolier, D. Louër and G. Férey, J. Am. Chem. Soc., 2002, 124, 13519-13526; (c) A. Schneemann, V. Bon, I. Schwedler, I. Senkovska, S. Kaskel and R. A. Fischer, Chem. Soc. Rev., 2014, 43, 6062-6096; (d) H. Sato, W. Kosaka, R. Matsuda, A. Hori, Y. Hijikata, R. V. Belosludov, S. Sakaki, M. Takata and S. Kitagawa, Science, 2014, 343, 167-170; (e) J. A. Mason, J. Oktawiec, M. K. Taylor, M. R. Hudson, J. Rodriguez, J. E. Bachman, M. I. Gonzalez, A. Cervellino, A. Guagliardi, C. M. Brown, P. L. Llewellyn, N. Masciocchi and J. R. Long, Nature, 2015, 527, 357.

4 B. Chen, C. Liang, J. Yang, D. S. Contreras, Y. L. Clancy, E. B. Lobkovsky, O. M. Yaghi and S. Dai, Angew. Chem., Int. Ed., 2006, 45, 1390-1393.

5 (a) T. Jacobs, G. O. Lloyd, J.-A. Gertenbach, K. K. Müller-Nedebock, C. Esterhuysen and L. J. Barbour, Angew. Chem., Int. Ed., 2012, 124, 4997-5000; (b) E. J. Carrington, I. J. Vitorica-Yrezabal and L. Brammer, Acta Crystallogr., Sect. B: Struct. Sci., Cryst. Eng. Mater., 2014, 70, 404-422; (c) R. Vaidhyanathan, S. S. Iremonger, G. K. H. Shimizu, P. G. Boyd, S. Alavi and T. K. Woo, Science, 2010, 330, 650-653.

6 S. Takamizawa, E. Nakata, H. Yokoyama, K. Mochizuki and W. Mori, Angew. Chem., Int. Ed., 2003, 115, 4467-4470.

7 (a) T. K. Maji, G. Mostafa, R. Matsuda and S. Kitagawa, J. Am. Chem. Soc., 2005, 127, 17152-17153; (b) J.-P. Zhang and X.-M. Chen, J. Am. Chem. Soc., 2009, 131, 5516-5521.

8 C. S. Yoo, H. Kohlmann, H. Cynn, M. F. Nicol, V. Iota and T. LeBihan, Phys. Rev. B: Condens. Matter Mater. Phys., 2002, 65, 104103.

9 S. Takamizawa, E. Nataka, T. Akatsuka, R. Miyake, Y. Kakizaki, H. Takeuchi, G. Maruta and S. Takeda, J. Am. Chem. Soc., 2010, 132, 3783-3792.

10 (a) S. Bourrelly, P. L. Llewellyn, C. Serre, F. Millange, T. Loiseau and G. Férey, J. Am. Chem. Soc., 2005, 127, 13519-13521; (b) T. M. McDonald, W. R. Lee, J. A. Mason, B. M. Wiers, C. S. Hong and J. R. Long, J. Am. Chem. Soc., 2012, 134, 7056-7065; (c) A. M. Plonka, D. Banerjee, W. R. Woerner, Z. Zhang, J. Li and J. B. Parise, Chem. Commun., 2013, 49, 7055-7057.

11 P. L. Llewellyn and G. Maurin, C. R. Chim., 2005, 8, 283-302. 